\title{
Primer registro de nido de Perdiz Austral (Tinamotis ingoufi Oustalet 1890) en Chile. Nuevas observaciones sobre su hábitat, dieta y reproducción en la región de Magallanes
}

\author{
First record of patagonian tinamou nest \\ (Tinamotis ingoufi oustalet 1890) in Chile. New observations on \\ their habitat, diet and reproduction in the Magellan region \\ Ricardo Matus ${ }^{1}$, Luis Burgos ${ }^{2}$, Santiago Imberti ${ }^{3}$, Osvaldo Vidal ${ }^{4}$, \\ Sebastian Saiter ${ }^{5}$, Claudio Vidal ${ }^{5}$, Enrique Couve ${ }^{5} \&$ Rodrigo Tapia $^{5}$
}

Las perdices (Tinamidae: Struthioniformes) son un grupo de aves que comprenden 5 especies presentes en el territorio chileno (del Hoyo et al. 1992, Couve et al. 2016), con una amplia presencia en matorrales estepáricos desde el nivel del mar hasta los 5.000 msnm (IUCN, 2017). Son aves herbivoras, y aunque algunas especies son abundantes como la Perdiz chilena (Nothoprocta perdicaria Kittlitz 1830), no existe información detallada respecto de la dieta de cada especie (Del Hoyo et al. 1992), y la información disponible sobre historia natural es anecdótica y dispersa. Por ejemplo, Pozzi (1923) y Goodall et al. (1951), indican que la Perdiz austral se alimenta de la chaura (Gaultheria pumila [L. f.] Hook. \& Arn. 1834) y frutos maduros del calafate (Berberis microphylla G. Forst. 1787), pero probablemente se omiten muchos ítems alimentarios de la dieta. Mas aún, otros aspectos como etológicos, reproductivos y de selección de hábitat son casi desconocidos (Couve et al. 2016) y requieren ser dilucidados.

Goodall et al. (1951), indican que la Perdiz austral está presente en las regiones de Aysén y Magallanes, sin hacer mención sobre sus abundancias, salvo el comentario de congregaciones de 30 a 40 individuos en otoño. Posteriormente Markham (1971), indica que se trata de un ave residente presente en la estepa centro oriental de la Provincia de Última Esperanza. Venegas y Jory (1979) por otra parte, indican que la presencia de la Perdiz austral es regular durante todo el año en el área de la estepa de la región de Magallanes (Pali Aike, Gallegos Chico, Monte Aymond y Punta Dungeness). Sin embargo, la única evidencia de su presencia en la región se basa en la colecta de dos ejemplares capturados por S. Díaz en el año 1975 (CZIP A448, CZIP A449 [Colección Zoológica Instituto de la Patagonia]), junto con el registro de dos ejemplares reportados por $\mathrm{H}$. Rohers en julio de 1986 en la comuna de San Gregorio (Venegas \& Sielfeld, 1998). Adicionalmente, existen 2 registros más recientes para las localidades de Bahía Posesión y Bahía Punta Delgada, Magallanes (Barros \& Schmitt 2008; Barros \& Schmitt, 2015). A pesar de los asistemáticos, pero intensos esfuerzos realizados por observadores de aves en el área, no se han reportado mayores antecedentes sobre esta especie en la región de Magallanes durante los últimos 40 años. Los registros realizados por S. Imberti en Punta Dungeness en enero de 2004, C. González en diciembre de 2007 y luego por un grupo de investigadores norteamericanos

Centro de Rehabilitación de Aves Leñadura, Kilómetro 7 Sur. $\bowtie$ rmatusn@gmail.com

2 Hostal San Gregorio, Don Bosco 190, Villa Punta Delgada, Magallanes, Chile. hostal_comidas@hotmail.com

3 Asociación Ambiente Sur, Carlos Gardel 389, Río Gallegos, Santa Cruz, Argentina. santiagoimberti@ambientesur.org.ar

4 Laboratorio de Botánica, Instituto de la Patagonia, Universidad de Magallanes, Avenida Bulnes 01890 Punta Arenas, Magallanes, Chile. osvaldo.vidal@umag.cl

5 Far South Expeditions, Manuel Señoret 610, Punta Arenas, Magallanes, Chile.

cvidal@farsouthexpeditions.com 
en diciembre de 2008 en el área de la Primera Angostura (Barros \& Schmitt, 2015), entregaron nuevos indicios, o al menos registros más recientes, sobre su presencia en la región de Magallanes. Respecto de su reproducción, en Chile no existen antecedentes en la literatura disponible actualmente. En Argentina se distribuye desde el norte de la provincia de Chubut, aunque ocasionalmente alcanzando a Río Negro, hasta el extremo sur de la provincia de Santa Cruz (Povedano \& Bisheimer, 2016). Ocupa todos los ambientes de estepa con preferencia a zonas que contengan parches de Mata Negra (Mulguraea tridens [Lag.] OLearly \& Peralta, 2009), desde el nivel del mar hasta los $1220 \mathrm{~m}$ en las mesetas de altura y hasta la zona de transición con el bosque andino (Darrieu et al. 2008; Imberti, 2003; Imberti, 2005; Roesler et al. 2014). Durante la época reproductiva es observada en grupos de tres individuos y durante el invierno forma agregaciones de hasta 40-50 individuos, generalmente más cerca de la costa, aunque esto puede deberse a una sub-observación (Pozzi, 1923; Imberti, 2003, Imberti, 2005; Roesler et al. 2014). La nidificación en Argentina comienza en septiembre-octubre cuando las vocalizaciones se escuchan con mayor frecuencia, y la puesta $e$ incubación ocurre entre noviembre-diciembre con los pichones eclosionando a fines de diciembre o en enero (Pozzi, 1923; Imberti, 2003; Roesler et al. 2014). En Chile no existen antecedentes

Esta nota tiene como fin recopilar las observaciones de Perdiz austral realizadas en la región de Magallanes, Chile entre julio de 2013 y agosto de 2017, añadiendo información más detallada sobre el hábitat donde se realizaron los registros y también sobre los eventos puntuales de dieta y reproducción de esta especie cuyo estado de conservación en nuestro país es Rara según el Reglamento de la Ley de Caza, y está clasificada en categoría de Preocupación Menor (Least Concern) según IUCN (IUCN, 2017).

\section{HÁBITAT}

En los últimos ocho años se han realizado nuevos registros (particularmente fotográficos), por parte de observadores de aves aficionados en sectores similares a los descritos por Venegas y Jory (1979). Se presentan los avistamientos realizados en los últimos 20 años (Tabla 1), con el fin de caracterizar el tipo de hábitat que ocupa la especie. De esta manera un total de 17 avistamientos de la especie se han realizado entre 2007 y 2017. Según estas observaciones los grupos registrados varian entre los 1 y 9 individuos. Uno de los autores de esta contribución (Luis Burgos), encontró un nido de estas aves en el mes de octubre de 2013 (Fig. 1). El hábitat usado por la Perdiz Austral en el área de los registros corresponde al de la estepa patagónica, un hábitat árido, dominado por especies de pastos amacollados como el Coirón (Festuca gracillima Hook. f. 1947), donde las precipitaciones anuales alcanzan unos $300 \mathrm{~mm}$ (Pisano, 1977). Otras especies co-dominantes son arbustivas como el Senecio (Senecio patagonicus Phil. 1863) y la Paramela (Adesmia boronioides Hook. f. 1847), cuyos arbustos servirían para proteger los nidos (Fig. 1). Sin embargo, localmente la vegetación es un mosaico donde confluyen vegas y lagunas salobres, donde las especies dominantes cambian, haciendo el paisaje más heterogéneo y muy diverso en riqueza de especies a una escala de paisaje.

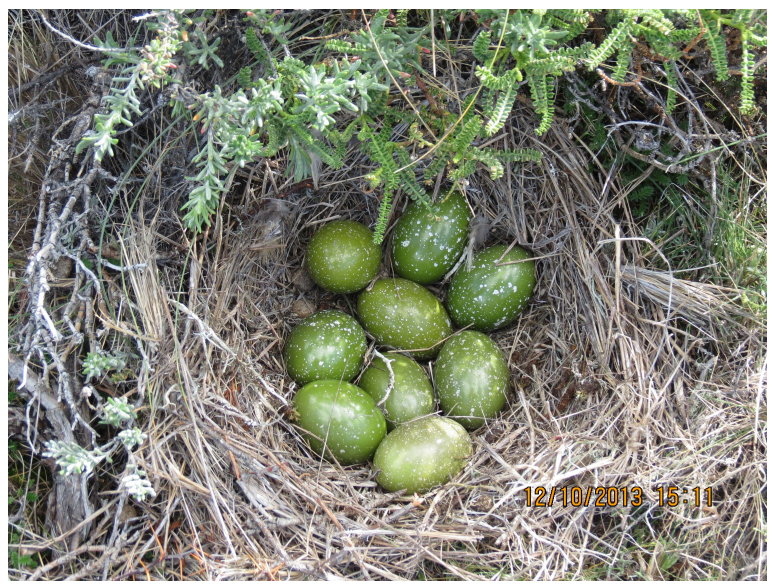

Fig. 1. Primer registro de nido de Perdiz austral T. ingoufi Oustalet 1890 en Chile. Contiene nueve huevos verdes con las diagnósticas pintas blancas. Nótese la cantidad de material para aislar los huevos del suelo (Fotografía: Luis Burgos).

\section{DIETA}

Un ejemplar hembra encontrada muerta el día 6 de junio de 2016 al lado de la carretera y colectado por Claudio Vidal, Enrique Couve y Rodrigo Tapia, fue presumiblemente golpeado por 
Tabla 1. Resumen de avistamientos y vocalizaciones de Perdiz austral registrados en la región de Magallanes.

\begin{tabular}{|c|c|c|c|c|}
\hline Fecha & $\mathrm{N}^{\circ}$ ejemplares & Localidad & Observador & Comentarios \\
\hline 18.12 .2007 & 3 & Bahía Posesión & C. González & $\begin{array}{c}\text { (Barros \& } \\
\text { Schmitt, 2008) }\end{array}$ \\
\hline 02.12 .2008 & 3 & Punta Delgada & Mark Peck & $\begin{array}{c}\text { (Barros \& } \\
\text { Schmitt, 2008) }\end{array}$ \\
\hline 22.07.2013 & 7 & Bahía Munición & L. Burgos & \\
\hline Verano 2013 & 9 & $\begin{array}{c}\text { Comuna de San } \\
\text { Gregorio }\end{array}$ & R. Crisóstomo & Cerca de la ruta \\
\hline 12.10 .2013 & 1 & Bahía Munición & L. Burgos & Fotografía de nido \\
\hline 07.11 .2015 & 1 & Pampa Larga & H. Cortez & Observado \\
\hline 04.06 .2016 & 1 & Bahía Santiago & C. Vidal & Atropello \\
\hline 19.09 .2016 & 2 & Bahía Santiago & $\begin{array}{c}\text { S. Saiter, } \\
\text { C. Pizarro y J. Rider }\end{array}$ & Fotografiadas \\
\hline 22.09.2016 & 3 & Bahía Santiago & F. Diaz & $\begin{array}{c}\text { Vocalizaciones cerca } \\
\text { de ruta } 255\end{array}$ \\
\hline 22.09.2016 & 6 & Bahía Santiago & R. Matus y R. Silva & Fotografiadas \\
\hline 23.09.2016 & 2 & Bahía Santiago & $\begin{array}{c}\text { S. Saiter, R. Tapia y } \\
\text { M. Figueroa }\end{array}$ & Vocalizaciones \\
\hline 04.11 .2016 & 2 & Punta Delgada & Fabrice Schmitt & Vocalizaciones \\
\hline 26.11 .2016 & 1 & Bahía Santiago & J. Paredes y J. Chamia & Vocalizaciones \\
\hline 18.02 .2017 & 8 & Bahía Santiago & S. Saiter y C. Pizarro & \\
\hline 25.02.2017 & 1 & Punta Delgada & J. Rider y M. Figueroa & \\
\hline 10.05 .2017 & 1 & Cuarto Chorrillo & Santiago Imberti & $\begin{array}{c}\text { Vocalizaciones cerca } \\
\text { de ruta } 255\end{array}$ \\
\hline 06.09 .2017 & 1 & Bahía Santiago & S. Saiter y C. Vidal & Vocalizaciones \\
\hline 31.08.2017 & 3 & Bahía Santiago & R. Matus y S. Saiter & \\
\hline 21.10 .2017 & 3 & Punta Dungeness & J. Paredes y J. Chamia & Fotografiados \\
\hline
\end{tabular}


un vehículo en movimiento. La piel del ejemplar fue preparada para ser conservada y el contenido del buche fue analizado para describir los ítemes dietarios. Los tejidos vegetales se conservaron en alcohol previo a su análisis, y se encontraron en condiciones adecuadas para su determinación taxonómica, principalmente debido que el buche solo tiene efectos mecánicos sobre las propiedades de los tejidos vegetales, no enzimáticos. Los fragmentos fueron contados y separados de acuerdo a su similitud, para determinar taxonómicamente las muestras. La determinación taxonómica de los fragmentos fue hecha mediante literatura morfológica (e.g. Correa, 1999; Moore, 1983), y comparaciones directas bajo lupa estereoscópica (modelo Olympus serie SZ), con ejemplares de referencia de la colección del herbario del Instituto de la Patagonia (HIP). La nomenclatura botánica sigue a Henríquez et al. (1995), actualizada según la base de datos Trópicos del Missouri Botanical Garden (2017). Posteriormente, los conteos fueron secados en horno de convección a $60^{\circ} \mathrm{C}$ por 48 horas para estimar peso seco de la biomasa por especie (modelo RadWag AS/ 220/C/2, precisión 0,1 mg).
Se encontró un total de 386 fragmentos de material vegetal atribuibles a 9 diferentes especies de plantas vasculares (Tabla 2, Fig. 2), las cuales sumaron un total de $1.27 \mathrm{gr}$ en peso seco. De todos los restos encontrados en el buche, aquellos de solo 2 especies (Adesmia lotoides Hook. f. 1847 y Suaeda patagonica Speg. 1897), concentran el $92,8 \%$ de la biomasa peso seco consumida (Tabla 2, Fig. 2), por lo que es posible inferir una alta selectividad de la dieta del individuo. Según estos resultados es posible presumir que el ejemplar colectado, es además, selectivo en el tipo de hábitat (estepa arbustiva), pues las plantas determinadas son, en su mayoría, características de ese tipo de hábitat.

\section{REPRODUCCIÓN}

El registro del día 12 de octubre de 2013 en Bahía Munición fue realizado por Luis Burgos y corresponde a la observación de un ejemplar de Perdiz austral levantando el vuelo desde un nido. Este nido consistía en una depresión en el suelo próximo

Tabla 2. Contenido de fragmentos (rastros) vegetales presentes en el buche del ejemplar de perdiz austral Tinamotis ingoufi, colectado en la localidad de San Gregorio (Magallanes), incluyendo información sobre el tipo de tejido vegetal colectado, número de fragmentos y peso seco de la biomasa por especie de cada una de las especies de plantas vasculares.

\begin{tabular}{|c|c|c|}
\hline Especie & Número de fragmentos & $\begin{array}{c}\text { Peso } \\
\text { biomasa seca (g) }\end{array}$ \\
\hline Adesmia lotoides Hook. f. & $294(76,2 \%)$ & $1,0453(82,13 \%)$ \\
\hline Arjona patagonica Hombr. \& Jacquinot ex Decne. & $1(0,3 \%)$ & $0,0051(0,40 \%)$ \\
\hline Azorella caespitosa Vahl & $10(2,6 \%)$ & $0,0116(0,91 \%)$ \\
\hline Galium richardianum (Gillies ex Hook. \& Arn.) Endl. ex Walp. & $1(0,3 \%)$ & $0,0007(0,06 \%)$ \\
\hline Hypochoeris aff. radicata (?) & $1(0,3 \%)$ & $0,0288(2,26 \%)$ \\
\hline Plantago uniglumis Pilg. & $5(1,3 \%)$ & $0,0099(0,78 \%)$ \\
\hline Poaceae indeterminada & $32(8,3 \%)$ & $0,0259(2,04 \%)$ \\
\hline Suaeda patagonica Speg. & $34(8,8 \%)$ & $0,1352(10,62 \%)$ \\
\hline Vicia magellanica Hook. f. & $8(2,1 \%)$ & $0,0102(0,80 \%)$ \\
\hline Suma & $386(100 \%)$ & 1,2727 (100\%) \\
\hline
\end{tabular}



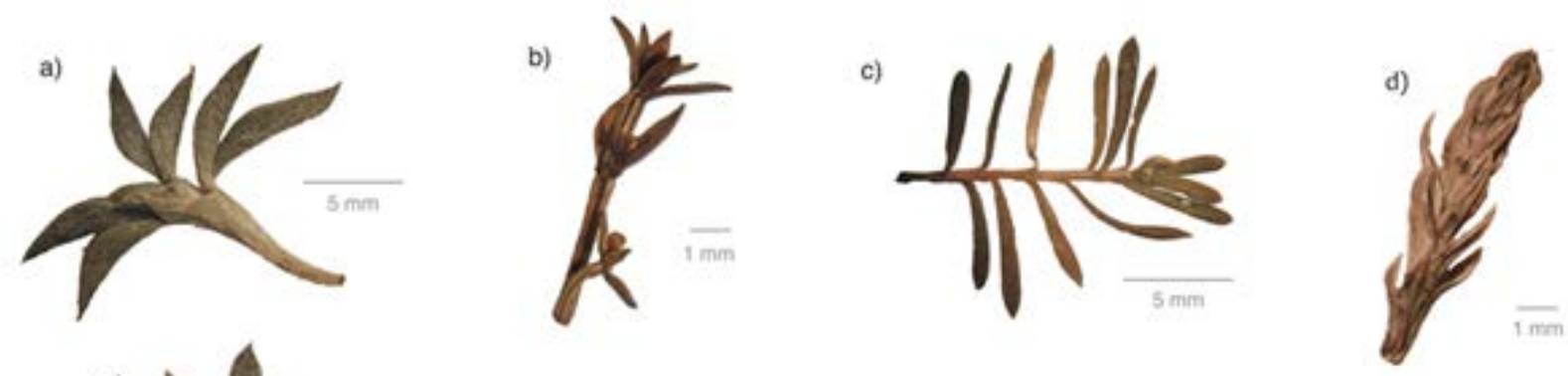

e)
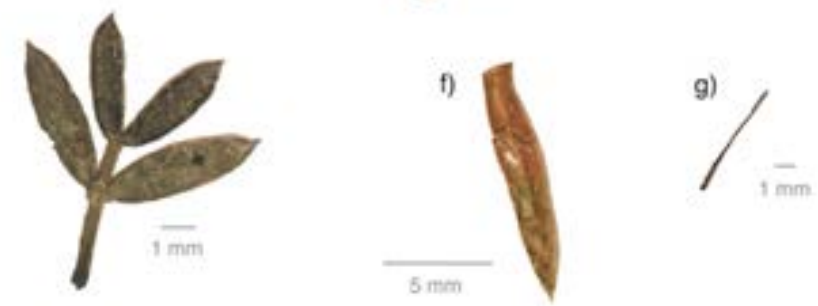

h)

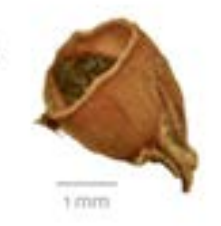

Fig. 2. Restos vegetales presentes en el buche del individuo de Perdiz Austral (Tinamotis ingoufi Oustalet 1890) colectado en el sector de San Gregorio (Magallanes): a) Adesmia lotoides (hojas y tallos), b) Galium richardianum (Gillies ex Hook. \& Arn.) Endl. Ex Walp. 1843 (hojas, tallos y flores axilares), c) Suaeda patagonica (hojas y tallos), d) Arjona patagónica Hombr. \& Jacquinot ex. Decne. 1853 (hojas y tallos), e) Vicia magellanica Hook. f. 1847 (hojas y pecíolo), f) Azorella caespitosa Cav. 1792 (lámina), g) graminoide atribuible a familia Poaceae (lámina linear) y, h) Plantago uniglumis Wallr. Ex Walp. 1843 (pixidio y semilla inserta).

a arbustos de Adesmia y Senecio cubierto por una capa de pastos secos usado como material aislante y contenía nueve huevos. Los huevos, de color verde, poseen pintas blancas que es una característica que diferencia a estos de los huevos de Perdiz copetona Eudromia elegans, otra especie de perdiz probable de observar en este tipo de hábitat y que tiene registros en zonas cercanas a la localidad de Posesión (J. Chamia com. pers.). En la ocasión no se tomaron medidas de los huevos y tampoco del nido. Este hallazgo se trataría de la primera evidencia de reproducción de la especie en la región de Magallanes y por lo tanto también en Chile.

Esta área de la comuna de San Gregorio, en la región de Magallanes, merece especial atención considerando los recientes registros de ambas especies de perdices. Esto con el fin de establecer su verdadero estatus a nivel regional y nacional a partir de un mayor esfuerzo de observación.

\section{DISCUSIÓN}

Un número significativo de los registros se realizaron en la localidad de bahía Santiago entre los años 2016 y 2017. Aparte de demostrar que la perdiz austral muestra una alta fidelidad a este sitio en particular, también se puede explicar como una respuesta del creciente interés de la comunidad local de observadores de aves y fotógrafos por esta especie de la cual existen pocas observaciones en nuestro país.

De esta manera, a partir del hallazgo de un nido y del análisis de contenido de buche de un ejemplar atropellado la presente nota entrega evidencia importante sobre aspectos desconocidos para la Perdiz austral en nuestro país sobre su reproducción y alimentación. Por otro lado, el número de observaciones realizadas permite inferir el tipo de hábitat y el tamaño de los grupos que se observan. Considerando que la zona donde se han realizado estas observaciones han sido prospectadas anteriormente por distintos observadores, la presencia de estas aves en la región de Magallanes en el periodo considerado en esta nota podrían ser el reflejo de un aumento en la dispersión desde el centro de su distribución en la estepa Argentina hacia los extremos, un fenómeno tal vez cíclico, que podría estar influenciado por las condiciones climáticas o inviernos menos rigurosos.

\section{AGRADECIMIENTOS}

Jaime Cárcamo del Laboratorio de Zoología del Instituto de la Patagonia (Universidad de Magallanes), proporcionó los números de registro 
de la Colección Zoológica (ZCIP). El Dr. Juan Carlos Aravena y el Dr. Rodrigo Villa de la Dirección de Programas Antárticos (DPA) de la Universidad de Magallanes facilitaron equipamiento para los análisis de contenidos del buche en el ejemplar colectado de T. ingoufi y a Christian Gonzalez B.

\section{LITERATURA CITADA}

Barros, R., \& F. Schmitt (2008). Resumen de avistamientos (noviembre 2007 - febrero 2008). La Chiricoca, 6, 20-27.

Barros, R., \& F. Schmitt (2015). Aves Raras en Chile, enero 2004-diciembre 2014. La Chiricoca, 20, 2-56

Correa, M. N. (1999). Flora patagónica. Buenos Aires: Colección científica del INTA.

Couve, E., Vidal, C. F., \& Ruiz, J. (2016). Aves de Chile, sus islas oceánicas y península antártica. FS Editorial.

Darrieu, C. A., Camperi, A. R., \& Imberti S. (2008). Avifauna (Non Passeriformes) of Santa Cruz province, Patagonia (Argentina): annotated list of species. Revista del Museo Argentino de Ciencias Naturales, 10, 111145.

Del Hoyo, J., Elliot, A., \& Sargatal, J. (1992). Handbook of the Birds of the World, Vol. 1. Barcelona: Lynx Edicions.

Goodall, J. D., Johnson A. W., \& Philippi, R. A. (1951). Las Aves de Chile: sus conocimientos y sus costumbres. Buenos Aires: Platt Establecimientos Gráficos S. A.

Henríquez, J. M., Pisano, E., \& Marticorena C. (1995). Catálogo de la flora vascular de Magallanes (XII Región), Chile. Anales del Instituto de la Patagonia, Serie Ciencias Naturales, 23, 5-30.

Imberti, S. (2003). Notes on the distribution and natural history of some birds in Santa Cruz and Tierra del Fuego provinces, Patagonia, Argentina. Cotinga, 19,15-24.

Imberti, S. (2005). Aves de los glaciares, inventario ornitológico del Parque Nacional Los Glaciares. Edición de Aves Argentinas y Administración de Parques Nacionales. Buenos Aires.

IUCN. (2017). The IUCN Red List of Threatened Species. Versión 2017-2. http://www. iucnredlist.org. Revisado el 6 de octubre de 2017.

Markham, B. J., (1971). Catálogo de los anfibios, reptiles, aves y mamíferos de la provincia de Magallanes. Punta Arenas: Instituto de la Patagonia.

Missouri Botanical Garden (2017). Tropicos.org. Revisado el 12 de octubre de 2017.

Moore, D. M. (1983). The Flora of Tierra del Fuego. Oswestry, Saint Louis, ix, 396 pp., 8 lám.

Pisano, E. (1977). Fitogeografía de FuegoPatagonia chilena I. Comunidades vegetales entre las latitudes 52 y $56^{\circ} \mathrm{S}$. Anales del Instituto de la Patagonia, 8, 121-250.

Povedano, H., \& Bisheimer, M. V. (2016). Aves terrestres de la Patagonia, Tierra del Fuego e islas del Atlántico Sur. Primera edición. Neuquén.

Pozzi, A. (1923). La perdíz de Santa Cruz (Patagonia) Tinamotis Ingoufi Oust. El Hornero, 3, 180-184.

Roesler, I., Imberti, S., Casañas, H., Hernández, P., Klavins, J., \& Pagano, L. (2014). Noteworthy records and natural history comments on rare and threatened bird species from Santa Cruz province, Patagonia, Argentina. Revista Brasileira de Ornitologia, 22(2), 189-200

Venegas, C., \& Jory, J. (1979). Guía de campo para las aves de Magallanes. Publicaciones del Instituto de la Patagonia. Serie Monografías 11.

Venegas, C., \& Sielfeld, W. (1998). Catálogo de los vertebrados de la región de Magallanes y Antártica chilena. Punta Arenas: Ediciones de la Universidad de Magallanes. 